\title{
The evolution of arch filament systems and moving magnetic features around a sunspot ${ }^{\star}$
}

\author{
Li Ma ${ }^{1,2}$, Wangping Zhou ${ }^{1,2}$, Guiping Zhou ${ }^{2}$, and Jun Zhang ${ }^{2}$ \\ ${ }^{1}$ CICAEET, School of Information Science \& Control, Nanjing University of Information Science \& Technology, 210044 Nanjing, \\ PR China \\ e-mail: wpzhou@nuist.edu.cn \\ 2 Key Laboratory of Solar Activity, National Astronomical Observatories, Chinese Academy of Sciences, 100012 Beijing, PR China
}

Received 27 August 2014 / Accepted 19 August 2015

\begin{abstract}
Context. Arch filament systems (AFSs) are usually considered as the chromospheric manifestations of the emerging flux regions (EFRs) seen in $\mathrm{H} \alpha$ observations. Moving magnetic features (MMFs) look similar to EFRs in magnetograms, but often appear in the decaying phase of an active region (AR) and behave differently from EFRs. A possible relation between AFS and MMF would be important for revealing a common mechanism for building up basic structures on the Sun.

Aims. Based on $\mathrm{H} \alpha$ and magnetic field observations with high spatial resolution, we study the evolution of MMFs around a sunspot, as well as their related AFSs from birth to death.

Methods. The multiwavelength observations from the New Vacuum Solar Telescope (NVST) and the Solar Dynamic Observatories (SDO) are co-aligned in the spatial and the temporal sense. MMFs appeared near the northern end of a light bridge (LB). Their related AFSs were carefully identified and traced from their appearance to disappearance based on $\mathrm{H} \alpha$, EUV data, and magnetograms.

Results. In the main sunspot of AR NOAA 11711 during April 1-4, 2013, many slow-speed MMFs with a polarity opposite to that of the sunspot appeared from the close vicinity of the northern end of a LB. Different from other smaller MMFs around the sunspot, these MMFs were always related to arch filaments and eventually formed AFSs with three twisting branches. The total flux involved in the AFSs was estimated to be about $2.7 \times 10^{21} \mathrm{Mx}$. The largest MMF "M1" evolved into a small pore that led to an intensity reduction in the continuum intensity images. The appearance and evolution of the AFSs near the sunspot seems to be controlled by MMFs emanating from the penumbra. Owing to continual magnetic cancellation between the MMFs and their surrounding opposite flux, the AFSs gradually disintegrated and finally disappeared.

Conclusions. The appearance and evolution of the AFSs near the sunspot seem to be controlled by these MMFs emanating from the penumbra.
\end{abstract}

Key words. sunspots - Sun: filaments, prominences - Sun: magnetic fields

\section{Introduction}

Magnetic fields are one of the basic characteristics on the Sun, and sunspots are the locations of intensive magnetic fields that trigger most of the solar eruptive activities. These solar activities have different hierarchies from large-scale structures, such as coronal mass ejection, to small-scale ones. Small-scale magnetic structures, such as emerging flux regions (EFRs) and moving magnetic features (MMFs), are considered as the basic magnetic elements that are associated with the growth and decay of a solar active region (AR). To find their manifestations in the chromosphere even in transition region, as well as the mechanisms for their appearance and disappearance, may contribute to a better understanding for solar activity.

EFRs were named by Zirin (1972) after their first notice by Waldmeier (1937). They are believed to contribute to the origin of an AR from the subphotosphere (e.g., Glackin 1975; Spadaro et al. 2004; Zuccarello et al. 2005). An EFR has magnetic flux with opposite polarity in magnetograms and appears as two small bright plages bridged by a cluster of parallel dark fibrils in the $\mathrm{H} \alpha$ filtergrams, which are called arch filament systems

* Movies are available in electronic form at http: //www . aanda.org
(AFSs; Bruzek 1967). A rising AFS is generally believed to trap photospheric material that drains down both ends as the arch rises (e.g., Bruzek 1969; Zirin 1987). Generally, the AFSs can be observed for several days, while individual arch filaments only exist a few tens of minutes. With the formation of new arch filaments, the old loops expand and can reach coronal heights (Spadaro et al. 2004). It has been suggested that these arch loops have heated up and expanded to hot coronal loops or at least that they play an important role in the formation of coronal loops (Kartashova 1992; Malherbe et al. 1998; Kurokawa \& Yoshimura 2000; Spadaro et al. 2004). Magnetic reconnections between the footpoints of AFSs and pre-existing ambient fields with opposite polarities can release a lot of energy and cause many eruptive activities, such as EUV brightenings or even flares (Longcope et al. 2005; Zuccarello et al. 2008; Tarr et al. 2014).

MMFs were first noticed by Sheeley (1969) in magnetograms and were defined by Harvey \& Harvey (1973). As a kind of basic magnetic elements on the Sun, MMFs play a key role in the decay phase of an AR (e.g., Criscuoli et al. 2012). They are described as streaming out of sunspot penumbrae and moving toward the outer boundary of the moat regions. MMFs can be categorized into three types: bipolar MMFs and the other two kinds of unipolar ones with their polarities the same as or opposite to those of the parent sunspots. The previous two types 
of MMFs have velocities of about $0.5-1 \mathrm{~km} \mathrm{~s}^{-1}$, and the last one had speeds in the range of $2-3 \mathrm{~km} \mathrm{~s}^{-1}$ (Shine \& Title 2001; Zhang et al. 2007). It has been suggested that bipolar MMFs are the manifestations of $\Omega$-shaped or U-shaped loops traveling along penumbral filaments, while unipolar MMFs are considered as the movement of single magnetic elements (Zhang et al. 2003; Zuccarello et al. 2009; Criscuoli et al. 2012). According to Thomas et al. (2002), MMFs should be the emerging effects of submerged penumbral flux tubes raised by convection.

Light bridges (LBs) are bright structures that appear in a sunspot and divide the umbra into many parts (Bray \& Loughhead 1964; Solanki et al. 1994; Solanki 2003). They are interpreted as field-free convection penetrating from the subphotosphere into the umbra. A LB usually has large magnetic inclinations and somewhat weaker magnetic fields (Lites et al. 1991; Rueedi et al. 1995; Leka 1997; Bellot Rubio et al. 2004). With magnetic fields decreasing and magnetic inclination increasing with height, a canopy structure appears above a LB (Jurčák et al. 2006). Such magnetic topology is supposed to be related to a variety of chromospheric activities (Asai et al. 2001; Louis et al. 2008, 2014a), including some recurrent and intermittent chromospheric ejections that are caused by magnetic reconnection between LB and umbra (Roy 1973; Asai et al. 2001; Bharti et al. 2007).

In this work, which is based on ground-based $\mathrm{H} \alpha$ observations with high-spatial resolution from the 1-m New Vacuum Solar Telescope (NVST) and multiwavelength observations from the Solar Dynamic Observatories (SDO), we study the activities in AR 11711, which includes the appearance of MMFs from the mid-inner penumbra in the natural prolongation of a LB, formation and disappearance of the MMFs-related AFSs, as well as a possible relationship with the LB. Section 2 describes the observations and data analysis. The association between the MMFs and the AFSs are analyzed in Sect. 3. Section 4 shows the summary and discussion, and Sect. 5 presents the conclusions.

\section{Observations and data reduction}

The primary $\mathrm{H} \alpha$ data are from the 1-m NVST of Fuxian Solar Observatory (FSO), operated by Yunnan Astronomical Observatory of China. The average seeing (Fried parameter, $r_{0}$ ) of FSO is about $10 \mathrm{~cm}$ (Liu et al. 2014). NVST provides two groups of main instruments, which are imaging instruments with 2D spectrometers and two grating spectrometers. As one of the most important facilities of FSO in China, the NVST is designed to observe the fine structure in the solar dynamical process, as well as magnetic field with high spatial and temporal resolution (Liu \& Xu 2011). During observations, three channels in the wavelengths of $\mathrm{TiO}, \mathrm{G}$ band, and $\mathrm{H} \alpha$ are used to image the Sun (Yang et al. 2014). The Lyot-filter used was centered on the $\mathrm{H} \alpha$ line at $6562.8 \AA$ and had a full bandpass width of $0.25 \AA$. Using a statistical algorithm, many images with a short exposure time $(\leq 20 \mathrm{~ms})$ were integrated into one frame with a certain field of view (FOV) called level-0 raw data. The level-0 raw data were improved to level-1 data by removing the dark current and applying a flat field correction and a lucky image selection algorithm (Liu et al. 2014).

The H $\alpha$ filtergrams $\left(141 \times 141 \operatorname{arcsec}^{2}\right)$ are registered on $850 \times 850$ pixels and have a temporal cadence of $12 \mathrm{~s}$ and a pixel size of 0.166 arcsec. Unfortunately, the ground-based $\mathrm{H} \alpha$ data for AR 11711 only cover the periods from 00:58-01:30 UT on 2 April and 05:11-07:30 UT on 3 April 2013. Using the approach described by Beck et al. (2007), the effective spatial resolution of the $\mathrm{H} \alpha$ images was estimated as about 0.5 arcsec on 2 April and 0.3 arcsec on 3 April 2013. To check the formation and evolution of the related $\mathrm{H} \alpha$ structures in the AR, EUV data from the Atmospheric Imaging Assembly (AIA; Lemen et al. 2012), longitudinal magnetic fields, and continuum intensity images from the Helioseismic and Magnetic Imager (HMI; Scherrer et al. 2012) on the satellite of SDO were also analyzed. The data from AIA and HMI have spatial resolutions of 0.5 and 0.6 arcsec/pixel and temporal cadences of $12 \mathrm{~s}$ and $45 \mathrm{~s}$, respectively. The multiwavelength data were aligned to the reference moment of 06:00 UT on 3 April 2013 by compensating for solar rotation. The EUV and HMI continuum intensity images from SDO were separately normalized to a reference value of $\mathrm{I}_{0}$ and $\mathrm{I}_{1}$, respectively, which are the averaged brightness and intensity of the EUV and continuum images in the quiet region of the FOV separately.

Figures $1 \mathrm{a}-\mathrm{c}$ show the co-aligned data for the $\mathrm{H} \alpha$ image with the full FOV of $141 \times 141 \operatorname{arcsec}^{2}$ from the NVST (Fig. 1a), the magnetogram (Fig. 1b), and continuum intensity image (Fig. 1c) from SDO/HMI at 01:25 UT on 2 April 2013. The subsets in the blue boxes of the top panels have a FOV of $58 \times 58 \operatorname{arcsec}^{2}$ and are correspondingly presented in Figs. 1d-f. The green dashed lines "A-B" in Fig. 1 indicate the long axis of an arch filament observed in the $\mathrm{H} \alpha$ observations (see Fig. 1d). As shown in the magnetogram of Fig. 1e, this arch filament connects the negative flux patch "A" and the positive flux patch "B", which is located in the penumbra of the sunspot in the AR NOAA 11711. The umbra of this sunspot was divided into three parts by two LBs, one of which (red arrow in Fig. 1f) is discussed in detail because of its possible relationship with MMFs and the MMFs-related AFSs studied in this work.

\section{Formation and evolution of AFSs}

The AR 11711 has been observed by NVST since 2 April 2013 when it was located close to the eastern edge of the Sun. In the $\mathrm{H} \alpha$ images, an arch filament (dashed lines "A-B" in Figs. 1d-i) connected the midpenumbra and the moat region of the sunspot (Figs. 1a-f). Transient $\mathrm{H} \alpha$ brightenings frequently happened near the northern end of the arch filament in the moat region, while many dark structures propagated along the long axis of the arch filament toward its southern endpoint in the vicinity of the LB (see the left panel of the movie attached Fig. 1). Some of these structures behaved as bright streaks and flashes, as well as resembling the chromospheric calcium jets (Louis et al. 2014a; see the yellow arrow in Fig. 1d).

In addition, in EUV $304 \AA$ images, bright structures were observed sweeping the periphery of the arch filament from the north to the vicinity of the LB (see Figs. 1g-i) and the middle panel of the movie attached to Fig. 1. The mass motion along the arch filament suggests a kind of connection between the moat region and the penumbra of the sunspot by an arched structure. In the FOV of the red box in Fig. 1b, we investigate the formation, evolution, and disappearance of the related arch filament, based on the combined multiwavelength data.

Figure 2 illustrates the appearance of the MMFs with the magnetic field of an opposite polarity and the formation of the MMFs-related AFSs. The temporal evolution of Fig. 2 is also shown in the corresponding animation online. From HMI magnetograms (Figs. 2a-d), four MMFs (M1-M4) were clearly identified. They all originated in the mid-penumbra, moved out 


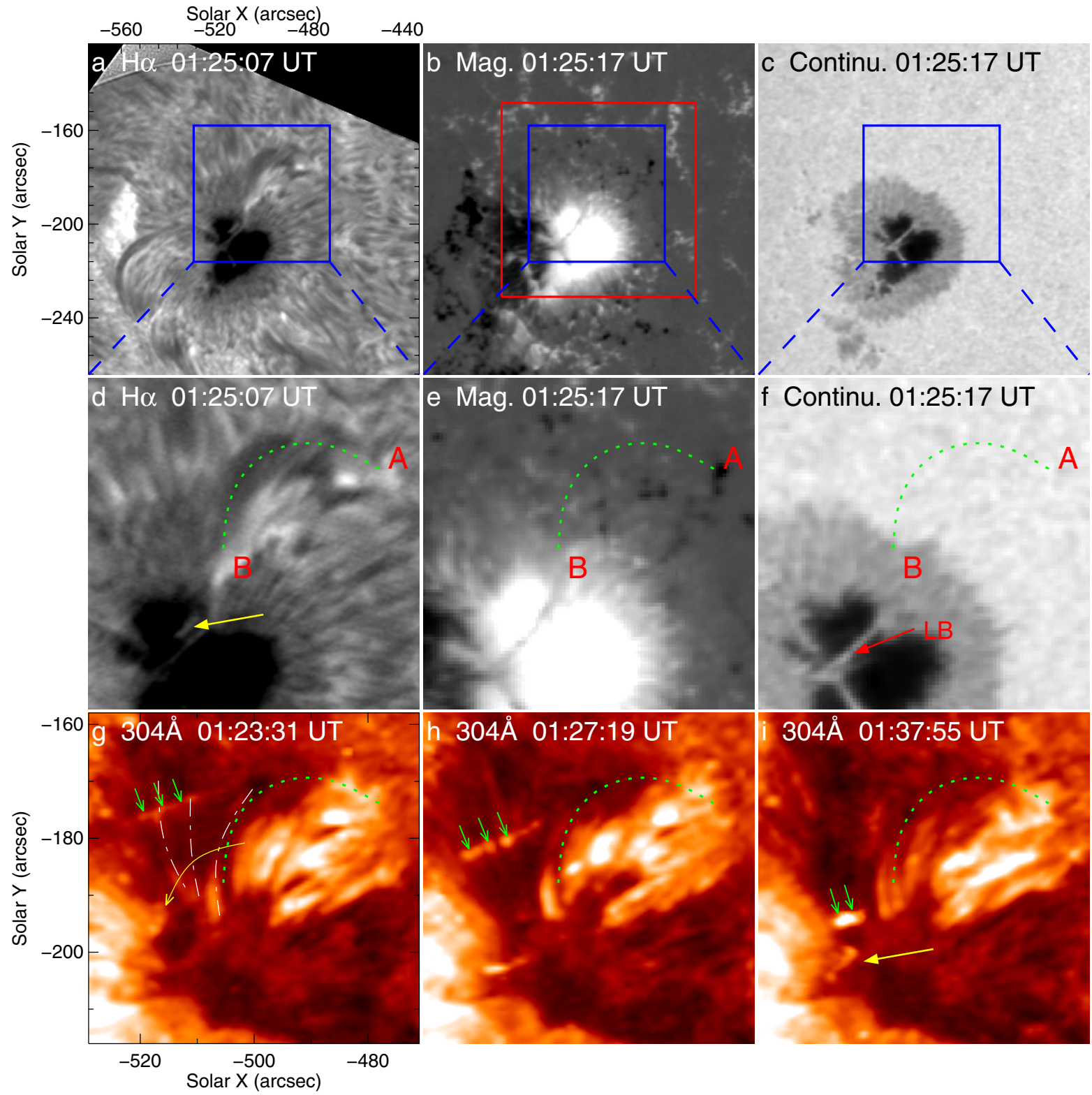

Fig. 1. Co-aligned images of the multiwavelength observations. Panels a)-f): the aligned $\mathrm{H} \alpha$, magnetogram, and continuum intensity images. Middle panels are the corresponding subsets of the top panels in the blue FOV. Panels $\mathbf{g}$ )-i): in $304 \AA$ images, bright mass motion (green arrows) swept the periphery (denoted by white dashed-dotted lines in panel g)) of the arch filament from the north to the south and even reached the very close vicinity of the LB (the yellow arrow in panel i)) in the blue FOV. A red box in panel b) denotes the FOV of subsequent Figs. 2-5. Green dotted lines "A-B" indicate the long axis of the arch filament. The red arrow in panel f) denotes the LB discussed in this work. An animation of the $\mathrm{H} \alpha, 304 \AA$, and HMI continuum intensity channels shown in this figure is available in the online edition.

of the sunspot, and finally disappeared at different places along the green dashed line shown in Fig. 2d, because of the rotation of the sunspot (see the continuum images in Figs. 2i-1). This sunspot first appeared at the eastern edge of the sun at about 11:30 UT on 30 March 2013. On 1 April (Fig. 2a), the flux patch of negative polarity denoted as M1 had already existed and acted as the northern magnetic end of an arch filament (see Fig. 2e). The other MMFs (e.g., M2-M4) were observed by AIA and HMI during their lifetimes and were carefully studied with emphasis on their evolution, as well as the formation and reconfiguration of their related AFSs.

As shown in Figs. 2a-d, MMFs M2-M4 appeared from almost the same location in the penumbra during the period of 1-2 April. The first appearance of these MMFs was in the natural extension of the related LB. Especially M4 appeared in the close vicinity of the LB's northern end. These MMFs slowly moved away from the sunspot. After they moved out of the penumbra, dark arch filaments can be seen to connect each of these MMFs and the penumbra, and they formed AFSs with three main branches (see F1, F2, and F3 in Figs. $2 \mathrm{~g}$ and $2 \mathrm{~h}$ ). The maximum magnetic flux of the four MMFs (M1-M4) was measured as $-7.7 \times 10^{20} \mathrm{Mx},-3.2 \times 10^{20} \mathrm{Mx},-8.4 \times 10^{19} \mathrm{Mx}$, and $-1.9 \times 10^{20} \mathrm{Mx}$, respectively. The total flux of the AFSs reached $2.7 \times 10^{21} \mathrm{Mx}$, if assuming equal positive and negative flux inside. Compared with the magnetic flux of the EFR-related AFSs (Spadaro et al. 2004; Zuccarello et al. 2008), the MMFsrelated AFSs studied here involved a modest amount of magnetic flux. These MMFs (M1, M2, and M3) cancelled out with their 


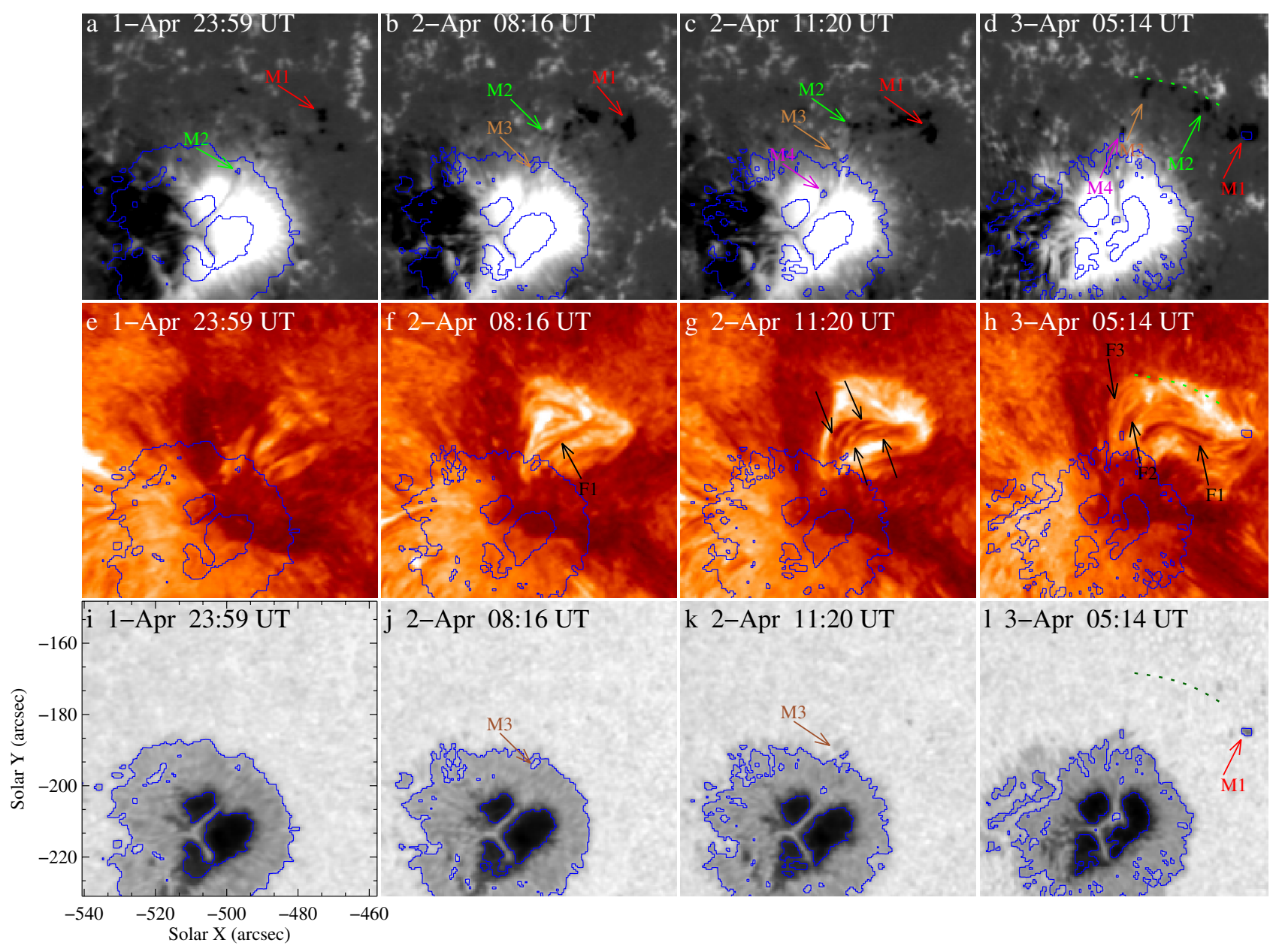

Fig. 2. MMFs and their related AFSs. Panels a)-d): HMI magnetograms showing the MMFs of negative polarity (M1, M2, M3, and M4 denoted by red, green, brown, and purple arrows) emerging from the penumbra of the sunspot. Panels e)-h): the MMFs related arch filaments (F1, F2, F3) in EUV $304 \AA$ images. Panels i)-l) are the corresponding continuum intensity images at each moment. MMFs are observed to frequently cancel with their surrounding positive flux in the place as denoted by dotted lines in panels $\mathbf{d}$ ), $\mathbf{h}$ ), and $\mathbf{l}$ ). Continuum intensity are contoured in each panel at the simultaneous moment with the value of $[0.3,0.8] \mathrm{I}_{1}$. An animation of the HMI magnetograms, $304 \AA$ and HMI continuum intensity channels shown in this figure is available in the online edition.

surrounding opposite flux (dotted curve in Fig. 2d), resulting in several EUV brightenings (see Fig. 2h).

The MMFs-related AFSs can be observed in lines forming both at higher and lower temperatures (see the movie attached to Fig. 3), such as H $\alpha$ (Fig. 3c), EUV $304 \AA$ (Fig. 3d), $211 \AA$ (Fig. 3e), and $131 \AA$ (Fig. 3f). At 06:05 UT on 3 April, the MMFs (M1, M2, and M3) arrived at the outer boundary of the moat. The largest MMF M1 evolved into a small pore that led to an intensity reduction in the continuum intensity images (see Fig. 3a). With the movements of converging and spinning for the MMFs, their related AFSs formed twisted structures and fan-shaped distributions (arrows in Figs. 3c-f). The multiple branches of the AFSs covered the area about $980 \operatorname{arcsec}^{2}$ with the maximum length and width of about 43 and 39 arcsec, repsectively. Each branch of the AFSs was made up of several bundles of twisted fibrils; for example, F1 exhibited a certain twisting (dashed curves in the black boxes).

A time-space plot (see Fig. 4c) from the HMI magnetograms was used to estimate the speed of M4 along its path "a-b" (blue dashed lines in Figs. 4a, b) from 11:19 UT on 2 April (Fig. 4a) to $14: 39$ UT on 3 April (Fig. 4b). The average moving velocity of M4 was estimated as about $0.24 \mathrm{~km} \mathrm{~s}^{-1}$ by a linear fitting. A lot of smaller MMFs (e.g., blue arrow in Fig. 4c) also shared a similar emergence.

\section{Magnetic cancellation, filament reconstruction, and disappearance of the AFSs}

When the MMFs reached the outer boundary of the moat, magnetic cancellation occurred between the opposite magnetic flux, and their related counterparts in the chromosphere and transition regions were gradually destroyed. Figure 5 illustrates that the MMFs disappeared owing to continual magnetic cancellation (see panels a-d and the left panel of the movie attached to Fig. 5), while EUV brightenings occurred among their related branches in $304 \AA$ images (see panels e-1 and the right panel with the movie attached to Fig. 5). As an example, MMF M3 existed at 11:12 UT on 3 April in Fig. 5a and disappeared at about 12:52 UT in Fig. 5b. During this period, eruptive activity happened among the AFS related to M3 and led to an enhanced EUV brightening with a two-ribbon flare (Fig. 5f) and a mass ejection (arrow in Fig. 5g) in $304 \AA$ images. In a similar way, MMFs M1, M2, and M4 experienced magnetic cancellation with northern environmental opposite flux and disappeared in 


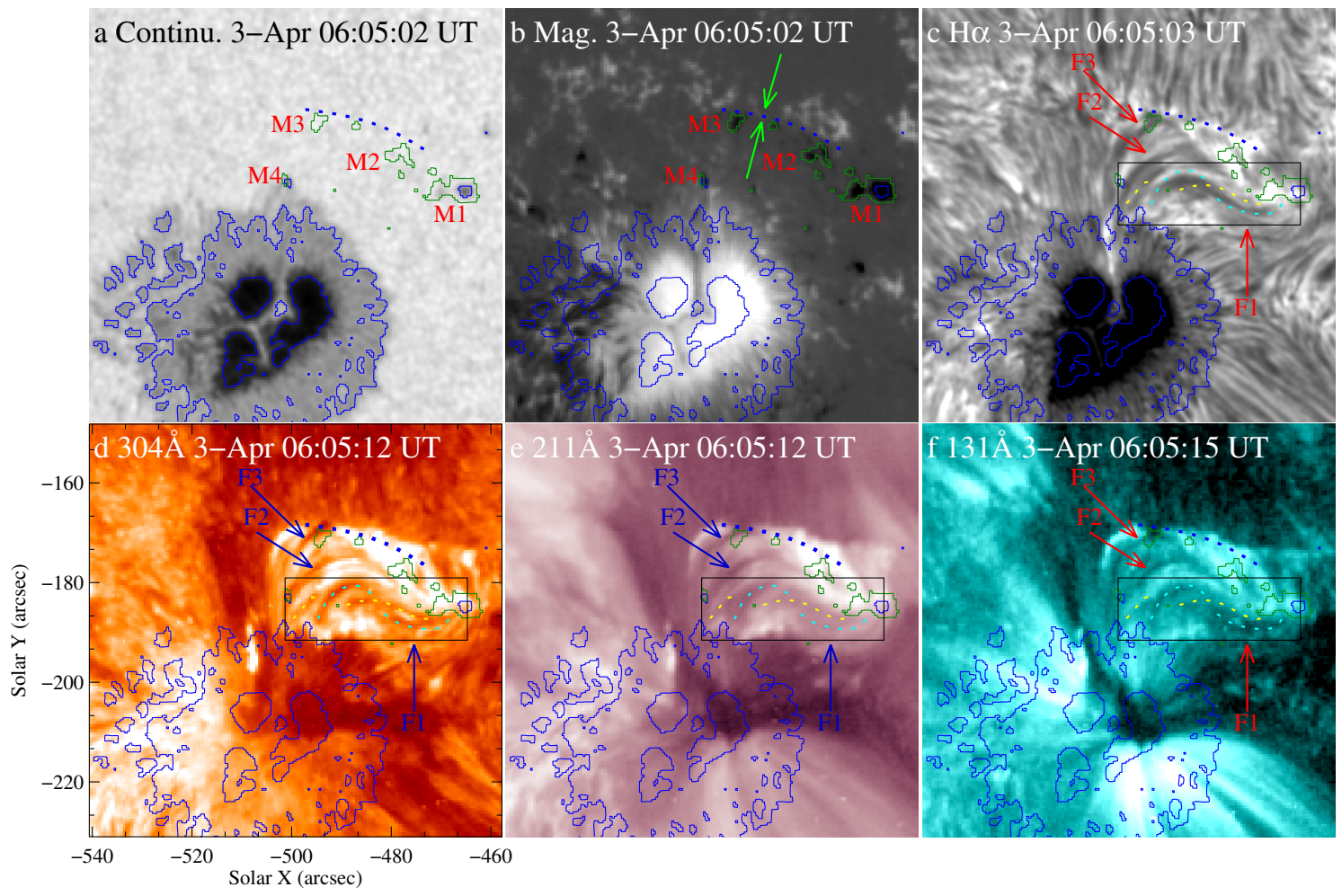

Fig. 3. MMFs and the related AFSs in multiwavelength observations at 06:05 UT on 3 April 2013. Panel a): continuum-intensity image showing the positions of the MMFs (M1, M2, M3, and M4) relative to the sunspot. Panel b): MMFs in the magnetogram image. Panels c)-f): the related filaments (F1, F2, F3) in the observations of H $\alpha$ (panel c)), EUV 304 (panel d)), 211 (panel e)), and 131 (panel f)) $\AA$. The fibrils in F1 showed some twisting (see dotted curves in the black boxes). The blue and green contours denote the continuum intensity and magnetic flux density with the values of $[0.3,0.8] \mathrm{I}_{1}$ and $[-110] \mathrm{G}$, respectively. An animation of the HMI continuum intensity, HMI magnetograms, H $\alpha, 304 \AA$, $211 \AA$, and $131 \AA$ channels shown in this figure is available in the online edition.
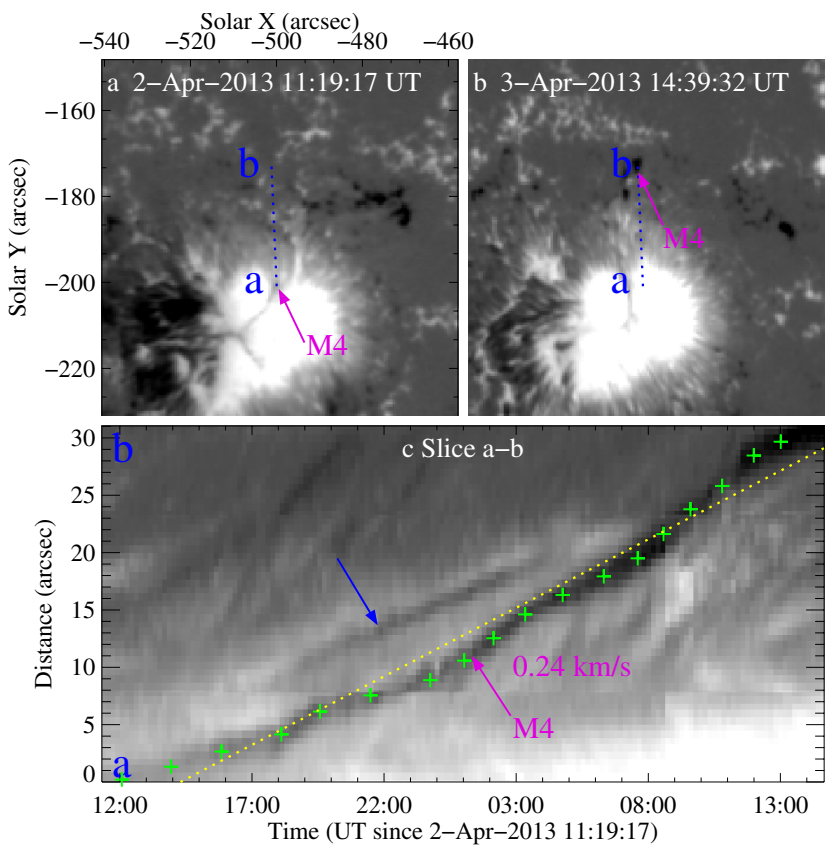

Fig. 4. Time slice along " $a-b$ " in magnetograms to estimate the velocity of M4. The slice line was chosen to connect initial and final position of M4 in panels a)-b). Panel c) was the time-space slice plot along " $a-b$ ". The average velocity of M4 was estimated as about $0.24 \mathrm{~km} \mathrm{~s}^{-1}$ by a linear fitting.

their respective circles (see Figs. $5 c-d$ ). Their related branches were gradually destroyed by EUV eruptive activities, such as
EUV brightenings and the ejection of dark structures shown in Figs. 5i-1.

Apart from the MMFs described, other negative-polarity flux was also observed to originate in the extension of the LB and formed a dark flux ribbon at 21:58 UT 3 April (cyan arrow in Fig. 5c). This flux ribbon also moved outward from the sunspot and cancelled with the positive fields near the outer boundary of the moat. With the first appearance of M1, M2, M3, and M4 before 1 April at 23:59 UT on 1 April at 08:16 UT and 11:20 on 2 April, the MMFs had lifetimes of approximately $>63 \mathrm{~h}$ (M1), $49 \mathrm{~h}$ (M2), $28 \mathrm{~h}$ (M3), and $44 \mathrm{~h}$ (M4), respectively.

To show the flux evolution of the studied MMFs, Fig. 6 presents the temporal evolution of the total, unsigned negative and positive magnetic flux and EUV brightness in $304 \AA$ observations in the region as denoted by the white rectangles in Figs. 5d and 1 from 01:30 UT on 3 April to 16:18 UT on 4 April. The bottom red line represents the average brightness $\mathrm{I}_{0}$ in a quiet region of the $304 \AA$ channel. From 01:30 UT to 22:30 UT on 3 April, all the three kinds of flux decreased quickly owing to magnetic cancellation. Accompanying the continual magnetic cancellation, several EUV brightenings occurred in this region and led to many spikes in the intensity curve. Two events of intense EUV eruptions happened at 11:15 and 21:58 UT on 3 April, whose brightness reached up to $7.4 \mathrm{I}_{0}$ and $2.9 \mathrm{I}_{0}$, as shown in Figs. 5f and $\mathrm{i}$. The average flux cancellation rate between 01:30 UT and 22:30 UT on 3 April is about $2.0 \times 10^{16} \mathrm{Mx} \mathrm{s}^{-1}$. During the first brightening from 10:00 UT to 11:30 UT, there was also a small peak in the GOES X-ray flux, the flux cancellation rate is estimated to be 


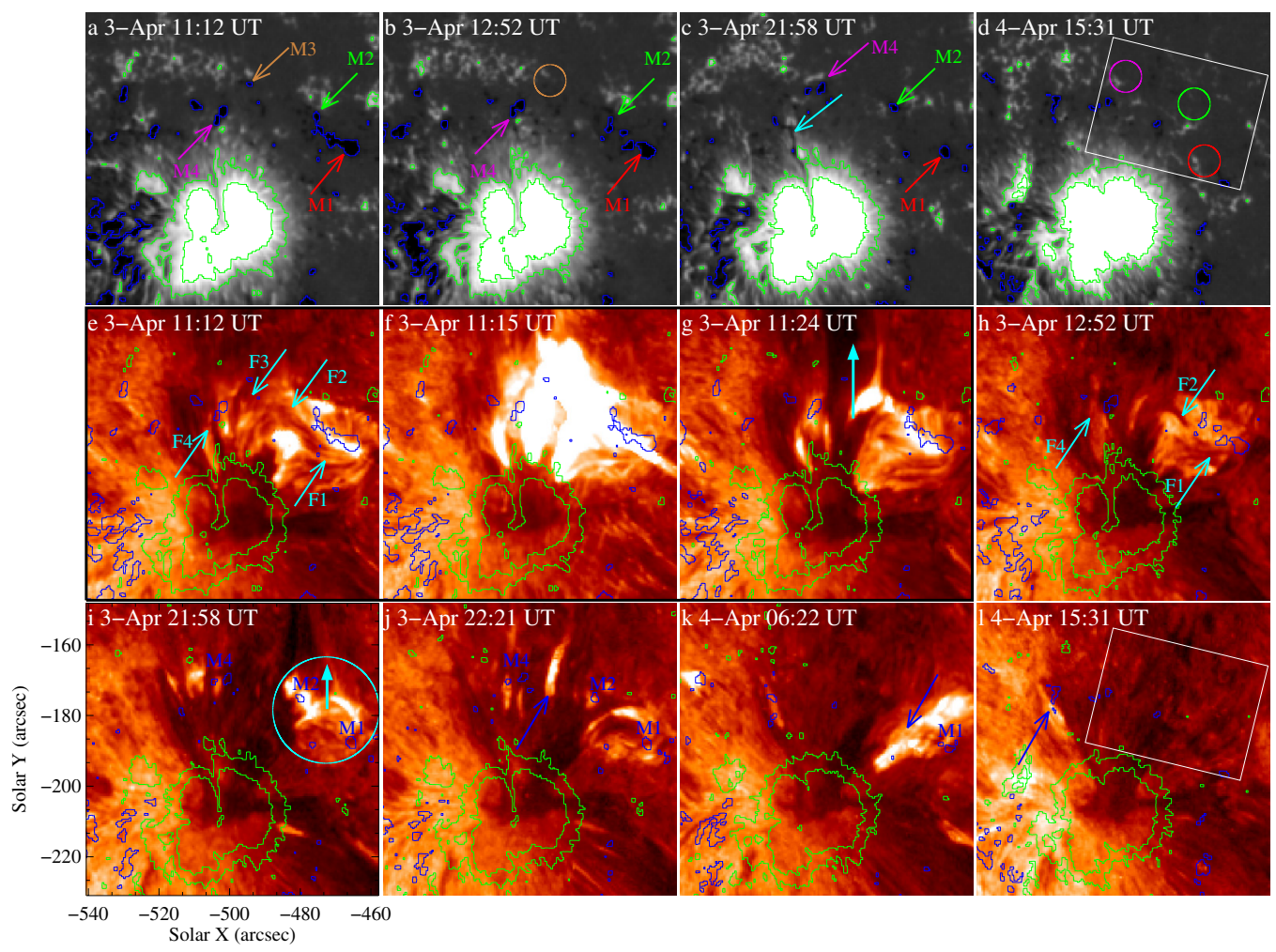

Fig. 5. Disappearance of the MMFs in magnetograms (panels a)-d)) and evolution of the related AFSs in EUV $304 \AA$ images (panels e)-l)). In panels (a)-b)), the negative flux of M3 was cancelled and disappeared in the brown circled area. Its related AFS F3 (see panel e)) was almost destroyed by experiencing a series of EUV brightening or EUV flare (panel f)) and mass ejections (see the arrow in panel g)). In a similar way, the disappearance of M1, M2, M4 was shown in panels c)-d), and their related AFSs are illustrated in panels i)-l). The magnetic flux is contoured with the value of $[-110,500,1000] \mathrm{G}$ with a blue color for negative polarity and green for positive ones. An animation of the HMI magnetograms and $304 \AA$ channels shown in this figure is available in the online edition.

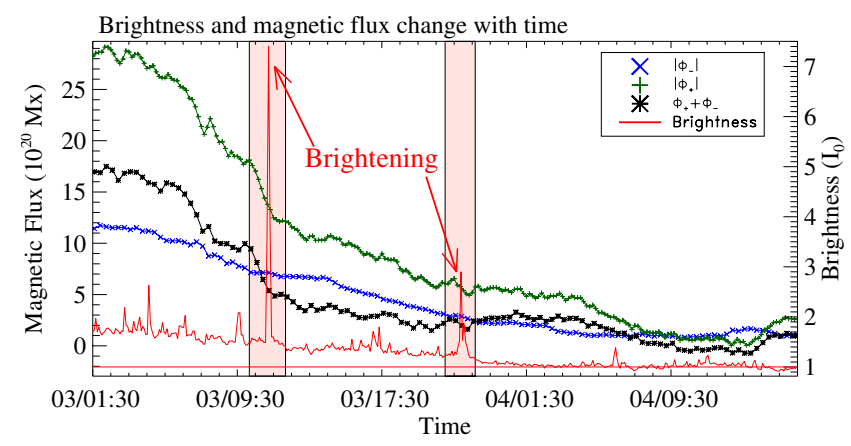

Fig. 6. Temporal evolution of magnetic flux and brightness in the EUV $304 \AA$ channel (see the red curve) in the white rectangles of Figs. $5 d$ and 1 from 01:30 UT on 3 April 2013 to 16:18 UT on 4 April 2013. The black profile indicates the evolution of total magnetic flux, while the blue and green profiles denote the unsigned negative and positive magnetic flux. Two times of intense EUV eruptions are denoted by two arrows in the two vertical rectangles.

about $1.1 \times 10^{17} \mathrm{Mx} \mathrm{s}^{-1}$, which is five times higher than the average cancellation rate. After 22:30 UT on 3 April, there was only a small amount of negative magnetic flux which decreased slowly in this area. When most of the negative flux of the MMFs had cancelled, this region became quiet without transient EUV brightenings.

\section{Summary and discussion}

Combining $\mathrm{H} \alpha$ observations from NVST, magnetograms and EUV data from SDO, we studied the appearance and evolution of unipolar MMFs from the mid-inner penumbra, as well as the formation and reconfiguration of their chromospheric manifestations as AFSs. The results show that:

1. Unipolar MMFs continuously appeared from the mid-inner penumbra in the natural extension of a LB and moved toward the moat region of the sunspot. The average speed of the MMFs was estimated as about $0.24 \mathrm{~km} \mathrm{~s}^{-1}$. When the MMFs moved out of the penumbra, dark fibrils in EUV $304 \AA$ images were observed to connect these MMFs and the midinner penumbra. At a later phase in the evolution of the MMFs, dark fibrils gradually packed and twisted together along with the converging movements of the MMFs in the photosphere, and eventually formed AFSs.

2. When the MMFs reached the moat region, magnetic cancellation occurred between MMFs of negative polarity and their surrounding positive-polarity flux. At the same time, the AFSs underwent transient $\mathrm{H} \alpha$ and EUV brightenings, a two-ribbon EUV flare, and mass ejections. As a result, the AFSs were gradually destroyed.

In this work, we reported on AFSs-related to MMFs. These MMFs originated in the mid-penumbra and moved toward the moat region at a speed of $0.24 \mathrm{~km} \mathrm{~s}^{-1}$. They were slower, involved more flux, and lived much longer compared with the MMFs reported in Zhang et al. $(2003,2007)$. There were also many other smaller MMFs emanating from the sunspot and appearing in the FOV, but they did not lead to the formation of AFSs. Usually, AFSs connect bipolar emerging flux (Bruzek 1967, 1968; Weart 1970; Zhang et al. 2006), but the 
MMFs-related AFSs studied here connect MMFs and penumbra. One end of the AFSs with positive polarity seems to be restrained in the penumbra of the sunspot and could not be identified with the current observations. However, some similarities between the AFSs still exist related to MMFs and those related to EFRs. The AFSs related to MMFs had a maximum length of 43 and width of 39 arcsec, involved a total magnetic flux $\sim 10^{21} \mathrm{Mx}$, and had a lifetime of four days. These parameters are quite similar to that of AFSs related to EFRs (Bruzek 1967; Mandrini et al. 2002; Zuccarello et al. 2008). Besides, we found similar structures at the eastern extension of another LB, which also seem to be formed by similar MMFs, although the magnetic structures in that region were very complicated and were not fully included in the current FOV.

Our observations indicate a definable relationship between the LB and the MMFs, as well as the related AFSs: 1) The MMFs appeared from almost the same photospheric source region, which was in the natural northern extension of the LB. 2) When the related AFSs anchored the MMFs and their source near the LB, dark $\mathrm{H} \alpha$ and brightening EUV structures (see also Louis et al. 2014b) were observed to move along the AFSs inward to the close vicinity of the LB. 3) The LB kept an anticlockwise rotation along with the sunspot motion, which resulted in a fan-like shape of the AFSs because the MMFs appeared in the order of time (see Fig. 3). And 4) at some moments, parts of fibrils of the AFSs could be observed to almost extend into the northern end of the LB (see the "F1" in Fig. 3c). All these observations suggest that the AFSs near the sunspot are controlled by the MMFs emanating from the penumbra at the prolongation of the LB.

LBs were also found to be associated with many aspects of solar activity, such as surges (Asai et al. 2001), filaments (Rimmele 2008), small-scale chromospheric jets (Louis et al. 2014a), and coronal jets (Liu 2012). By analyzing the magnetic fields of a LB, Shimizu et al. (2009) found that highly twisted flux tubes with current were trapped by the LB canopy structure and were responsible for the chromospheric ejections. The relationship among the MMFs, the AFSs, and the LBs is still an open question. It would be worthy of being studied based on observations with much higher temporal and spatial resolutions.

\section{Conclusions}

In this work, a scenario about the formation and evolution of the AFSs related to unipolar MMFs was revealed. On the one hand, AFSs were found to anchor MMFs and the mid-inner penumbra. All these MMFs appeared from the penumbra at the natural extension of the LB, slowly moved to the northwest of the sunspot at the speed of about $0.24 \mathrm{~km} \mathrm{~s}^{-1}$, and were always related to arch filaments. Spinning and converging movements of the MMFs gradually packed and twisted the filaments and formed the AFSs with multiple branches. The AFSs covered an area of about $980 \operatorname{arcsec}^{2}$ with a maximum length of 43 and a width of 39 arcsec. On the other hand, magnetic reconnection between the MMFs and moat fields triggered EUV brightenings and mass ejections (see also Yang et al. 2013a,b) recurrently in the wavelengths of $\mathrm{H} \alpha$ and EUV $304 \AA$, and were said to play a key role in the disappearance of the MMFs and destruction of the AFSs. The total negative flux composed of these MMFs involved in the AFSs was about $1.4 \times 10^{21} \mathrm{Mx}$, which quickly decreased because of the cancellation and finally resulted in the disappearance of the AFSs. The appearance and evolution of the AFSs near the sunspot seem to be controlled by these MMFs emanating from the penumbra.
Acknowledgements. We are grateful to the anonymous referee for the constructive suggestions and great improvements to this work. The data we used are courtesy of NVST and SDO science teams. The work is supported by the National Natural Science Foundation of China (11322329, 10973019 , 11025315, 11221063, and 11303049), the Chinese Academy of Science Project KJCX2-EW-T07, the National Key Basic Research Science Foundation (G2011CB811403 and G2011CB811402), the Open Research Program of Key Laboratory of Solar Activity, National astronomical Observatories (KLSA201416) and the Strategic Priority Research Program - The Emergence of Cosmological Structures of the Chinese Academy of Sciences, Grant No. XDB09000000.

\section{References}

Asai, A., Ishii, T. T., \& Kurokawa, H. 2001, ApJ, 555, L65

Beck, C., Bellot Rubio, L. R., Schlichenmaier, R., Sütterlin, P. 2007, A\&A, 472, 607

Bellot Rubio, L. R., Balthasar, H., \& Collados, M. 2004, A\&A, 427, 319

Bharti, L., Rimmele, T., Jain, R., Jaaffrey, S. N. A., \& Smartt, R. N. 2007, MNRAS, 376, 1291

Bray, R. J., \& Loughhead, R. E. 1964, Sunspots, The International Astrophysics Series (London: Chapman Hall)

Bruzek, A. 1967, Sol. Phys., 2, 451

Bruzek, A. 1968, Structure and Development of Solar Active Regions, IAU Symp., 35, 293

Bruzek, A. 1969, Sol. Phys., 8, 29

Criscuoli, S., Del Moro, D., Giannattasio, F., et al. 2012, A\&A, 546, A26

Glackin, D. L. 1975, Sol. Phys., 43, 317

Harvey, K., \& Harvey, J. 1973, Sol. Phys., 28, 61

Jurčák, J., Martínez Pillet, V., \& Sobotka, M. 2006, A\&A, 453, 1079

Kartashova, L. G. 1992, Bull. Crim. Astrophys. Obs., 86, 124

Kurokawa, H., \& Yoshimura, K. 2000, Adv. Space Res., 25, 1825

Leka, K. D. 1997, ApJ, 484, 900

Lemen, J. R., Title, A. M., Akin, D. J., et al. 2012, Sol. Phys., 275, 17

Lites, B. W., Bida, T. A., Johannesson, A., \& Scharmer, G. B. 1991, ApJ, 373, 683

Liu, S. 2012, PASA, 29, 193

Liu, Z., \& Xu, J. 2011, ASI Conf. Ser., 2, 9

Liu, Z., Xu, J., Gu, B.-Z., et al. 2014, RA\&A, 14, 705

Longcope, D. W., McKenzie, D. E., Cirtain, J., \& Scott, J. 2005, ApJ, 630, 596

Louis, R. E., Bayanna, A. R., Mathew, S. K., \& Venkatakrishnan, P. 2008, Sol. Phys., 252, 43

Louis, R. E., Beck, C., \& Ichimoto, K. 2014a, A\&A, 567, A96

Louis, R. E., Balthasar, H., Kuckein, C., et al. 2014b, Astron. Nachr., 335 , 161

Malherbe, J. M., Schmieder, B., Mein, P., et al. 1998, Sol. Phys., 180, 265

Mandrini, C. H., Démoulin, P., Schmieder, B., Deng, Y. Y., \& Rudawy, P. 2002, A\&A, 391, 317

Rimmele, T. 2008, ApJ, 672, 684

Roy, J. R. 1973, Sol. Phys., 28, 95

Rueedi, I., Solanki, S. K., \& Livingston, W. 1995, A\&A, 302, 543

Scherrer, P. H., Schou, J., Bush, R. I., et al. 2012, Sol. Phys., 275, 207

Sheeley, N. R., Jr. 1969, Sol. Phys., 9, 347

Shimizu, T., Katsukawa, Y., Kubo, M., et al. 2009, ApJ, 696, L66

Shine, T., \& Title, A. 2001, in Encyclopedia of Astronomy and Astrophysics, ed. P. Murdin (London: Nature Publishing; and Bristol: IOP Publishing), 4, 3209

Solanki, S. K. 2003, A\&ARv, 11, 153

Solanki, S. K., Montavon, C. A. P., \& Livingston, W. 1994, A\&A, 283, 221

Spadaro, D., Billotta, S., Contarino, L., Romano, P., \& Zuccarello, F. 2004, A\&A, 425, 309

Tarr, L. A., Longcope, D. W., McKenzie, D. E., \& Yoshimura, K. 2014, Sol. Phys., 289, 3331

Thomas, J. H., Weiss, N. O., Tobias, S. M., \& Brummell, N. H. 2002, Nature, 420, 390

Waldmeier, M. 1937, Astrophysics, 14, 91

Weart, S. R. 1970, ApJ, 162, 987

Yang, L., He, J., Peter, H., et al. 2013a, ApJ, 770, 6

Yang, L., He, J., Peter, H., et al. 2013b, ApJ, 777, 16

Yang, S., Zhang, J., Liu, Z., \& Xiang, Y. 2014, ApJ, 784, L36

Zhang, J., Solanki, S. K., \& Wang, J. 2003, A\&A, 399, 755

Zhang, J., Ma, J., \& Wang, H. 2006, ApJ, 649, 464

Zhang, J., Solanki, S. K., Woch, J., \& Wang, J. 2007, A\&A, 471, 1035

Zirin, H. 1972, Sol. Phys., 22, 34

Zirin, H. 1987, Sol. Phys., 114, 239

Zuccarello, F., Battiato, V., Contarino, L., et al. 2005, A\&A, 442, 661

Zuccarello, F., Battiato, V., Contarino, L., et al. 2008, A\&A, 488, 1117

Zuccarello, F., Romano, P., Guglielmino, S. L., et al. 2009, A\&A, 500, L5 